\title{
Non-local Thirring model at finite temperature
}

\author{
M.V. Manías ${ }^{\text {a,b }}$, C.M. Naón ${ }^{\text {a,b,1, }}$ M.L. Trobo ${ }^{\text {a,b }}$ \\ ${ }^{a}$ Depto. de Física. Universidad Nacional de La Plata. CC 67, 1900 La Plata, Argentina \\ b Consejo Nacional de Investigaciones Científicas y Técnicas, Argentina
}

Received 6 January 1998; accepted 21 April 1998

\begin{abstract}
We extend a recently proposed non-local and non-covariant version of the Thirring model to the finite-temperature case. We obtain a completely bosonized expression for the partition function, describing the thermodynamics of the collective modes which are the underlying excitations of this system. From this result we derive closed formulae for the free-energy, specific-heat, two-point correlation functions and momentum distribution, as functionals of electron-electron coupling potentials. (C) 1998 Elsevier Science B.V.
\end{abstract}

PACS: 11.10.-z; 11.15.-q

Keywords: Quantum field theory; Strongly correlated systems; Temperature correlations

\section{Introduction}

In recent years renewed interest has arisen in the study of low-dimensional field theories. In particular, research on the one-dimensional (1d) fermionic gas has been very active, mainly due to the actual fabrication of the so-called quantum wires [1]. One of the most interesting aspects of these systems is the possibility of having a deviation from the usual Fermi-liquid behavior. This phenomenon was systematically examined by Haldane [2] who coined the term Luttinger-liquid behavior to name this new physical situation in which the Fermi surface disappears and the spectrum contains only collective modes. Perhaps the simplest theoretical framework that presents this feature is the Tomonaga-Luttinger (TL) model [3], a many-body system of rightand left-moving particles interacting through their charge densities. In a recent series of papers [4] an alternative, field-theoretical approach was developed to consider this

\footnotetext{
${ }^{1}$ E-mail: naon@venus.fisica.unlp.edu.ar 
problem. In these works a non-local and non-covariant version of the Thirring model was introduced, in which the fermionic densities and currents are coupled through bilocal, distance-dependent potentials. This non-local Thirring model (NLT) contains the TL model as a particular case. Although it constitutes an elegant framework to analyze the 1d many-body problem, one serious limitation appears if one tries to make contact with quantum wires phenomenology. Indeed, one has to recall that the NLT has been formulated at zero temperature. This means, of course, that as it stands it cannot be used to study the Luttinger-liquid thermodynamics. The main purpose of this paper is to fill this gap. To this end we employ the well-known imaginary time formalism $[5,6]$ in order to obtain the finite-temperature version of the NLT model. In Section 2 we verify that the manipulations used to write the vacuum functional at $T=0$ in terms of a fermionic determinant, also work for the NLT at an arbitrary equilibrium temperature. Using the so-called decoupling technique at finite temperature [7] we obtain the partition function which describes the thermodynamics of the effective bosonic degrees of freedom (charge density and spin-density waves). In Section 3 we derive expressions for the Helmholtz free energy, the energy and the specific heat as functionals of the forward-scattering potentials. In Section 4 we compute the two-point fermionic correlation function and using this result, in Section 5 we obtain the formula for the momentum distribution. One of the most interesting aspects of these formulae is that being functionals of the potentials they could be employed as starting points in order to perform quantitative tests of different electron-electron couplings. Of course, these studies could be analytical or numerical, depending on the specific potentials to be considered. Finally, in Section 6, we summarize the main points of our investigation.

\section{The partition function}

In this section we study the two-dimensional non-local Thirring model [4] at finite temperature using the imaginary time formalism developed by Bernard [5] and Matsubara [6]. Our starting point is the Euclidean action given by

$$
S=\int_{\beta} d^{2} x \bar{\Psi} i \not \phi \Psi-\frac{g^{2}}{2} \int_{\beta} d^{2} x d^{2} y\left[J_{\mu}^{a}(x) V_{(\mu)}^{a b}(x-y) J_{\mu}^{b}(y)\right]
$$

where $\int_{\beta} d^{2} x$ means $\int_{0}^{\beta} d x^{0} \int d x^{1}$ and $\beta=1 / k_{\mathrm{B}} T$ with $k_{\mathrm{B}}$ the Boltzmann's constant and $T$ the temperature. The fermionic current is represented by $J_{\mu}^{a}=\bar{\Psi} \gamma_{\mu} \lambda^{a} \Psi$ with $\lambda^{0}=\frac{1}{2} I$, $\lambda^{j}=t^{j}, t^{j}$ being the $S U(N)$ generators normalized according to $\operatorname{tr}\left(t^{i} t^{j}\right)=\delta^{i j} / 2$. In order to make direct contact with a system of $1 \mathrm{~d}$ electrons, we introduce the Fermi velocity $v_{F}$ by defining the $\gamma_{\mu}$ matrices as

$$
\gamma_{0}=\left(\begin{array}{ll}
0 & 1 \\
1 & 0
\end{array}\right)
$$




$$
\gamma_{1}=\left(\begin{array}{cc}
0 & i v_{F} \\
-i v_{F} & 0
\end{array}\right)
$$

Of course, for $v_{F}=1$ one reobtains the set of matrices usually employed in $1+$ 1 QFT's. The functions $V_{(\mu)}^{a b}(x-y)$ are $N^{2} \times N^{2}$ matrices whose elements are the potentials describing electron-electron forward-scattering interactions. Let us anticipate that, although the procedure that follows works for arbitrary $N$, we shall be specially interested in the particular case $N=2$, which is the natural choice to consider spin- $\frac{1}{2}$ particles in this non-relativistic framework. To avoid confusion let us also note that no sum over repeated indices will be implied when a subindex $(\mu)$ is involved. In other words, the interaction between currents appearing in the action above reads

$$
J_{\mu}^{a}(x) V_{(\mu)}^{a b}(x-y) J_{\mu}^{b}(y)=J_{0}^{a}(x) V_{(0)}^{a b}(x-y) J_{0}^{b}(y)+J_{1}^{a}(x) V_{(1)}^{a b}(x-y) J_{1}^{b}(y)
$$

We shall consider the vacuum functional

$$
Z=N N_{F}(\beta) \int_{\text {antiper }} D \bar{\Psi} D \Psi e^{-S},
$$

where $N$ is an infinite $\beta$-independent constant while $N_{F}(\beta)$ is a $\beta$-dependent infinite factor that will be determined later on in this section. The functional integral in (2.5) must be extended over the paths with antiperiodicity conditions in the Euclidean time variable $x^{0}$ :

$$
\begin{gathered}
\Psi\left(x^{0}+\beta, x^{1}\right)=-\Psi\left(x^{0}, x^{1}\right), \\
\bar{\Psi}\left(x^{0}+\beta, x^{1}\right)=-\bar{\Psi}\left(x^{0}, x^{1}\right) .
\end{gathered}
$$

Exactly as one does in the usual (local and covariant) Thirring model, the fermionic quartic interaction can be eliminated by introducing auxiliary vector fields. In this way one can express the partition function in terms of a fermionic determinant. This, in turn, allows us to implement the path-integral approach to non-local bosonization [4], which we want to extend to the $T \neq 0$ case. To follow this procedure it is convenient to split $S$ in the form

$$
S=S_{0}+S_{\text {int }},
$$

where

$$
S_{0}=\int_{\beta} d^{2} x \bar{\Psi} i \not \phi \Psi
$$

and

$$
S_{\mathrm{int}}=-\frac{g^{2}}{2} \int_{\beta} d^{2} x J_{\mu}^{a} K_{\mu}^{a} .
$$

In this last expression $K_{\mu}^{a}$ is a new current defined as 


$$
K_{\mu}^{a}(x)=\int_{\beta} d^{2} y V_{(\mu)}^{a b}(x, y) J_{\mu}^{b}(y) .
$$

The partition function can now be written as

$$
Z=N N_{F}(\beta) \int_{\text {antiper }} D \bar{\Psi} D \Psi \exp \left\{-\int_{\beta} d^{2} x \bar{\Psi} i \phi \psi+\frac{g^{2}}{2} \int_{\beta} d^{2} x J_{\mu}^{a} K_{\mu}^{a}\right\}
$$

Now, we shall introduce a vector field $\tilde{A}_{\mu}$ in the form

$$
\int D \tilde{A}_{\mu}^{a} \delta\left(\tilde{A_{\mu}^{a}}-K_{\mu}^{a}\right) \exp \left\{\frac{g^{2}}{2} \int_{\beta} d^{2} x \tilde{A}_{\mu}^{a} J_{\mu}^{a}\right\}=\exp \left\{\frac{g^{2}}{2} \int_{\beta} d^{2} x K_{\mu}^{a} J_{\mu}^{a}\right\} .
$$

We represent the delta functional using a $\tilde{B}_{\mu}$-field as follows:

$$
\delta\left(\tilde{A}_{\mu}^{a}-K_{\mu}^{a}\right)=\int D \tilde{B}_{\mu}^{a} \exp \left\{-\int_{\beta} d^{2} x \tilde{B}_{\mu}^{a}\left(\tilde{A}_{\mu}^{a}-K_{\mu}^{a}\right)\right\} .
$$

We have to impose periodicity conditions for the bosonic $\tilde{A}_{\mu}$ and $\tilde{B}_{\mu}$-fields over the range $[0, \beta]$. Using now (2.12) and (2.13), the fermionic piece of the action can be written as

$$
S_{0}-\frac{g^{2}}{2} \int_{\beta} d^{2} x\left(\tilde{A}_{\mu}^{a} J_{\mu}^{a}+\frac{2}{g^{2}} \tilde{B}_{\mu}^{a} K_{\mu}^{a}\right)=\int_{\beta} d^{2} x \bar{\Psi}\left(i \not \partial-\frac{g^{2}}{2} \gamma_{\mu}\left(\tilde{A}_{\mu}^{a}+\bar{B}_{\mu}^{a}\right)\right) \Psi,
$$

where we have defined

$$
\bar{B}_{\mu}^{a}(x)=\frac{2}{g^{2}} \int_{\beta} d^{2} y V_{(\mu)}^{a b}(y, x) \tilde{B}_{\mu}^{b}(y) .
$$

For later convenience we shall invert (2.15) in the form

$$
\tilde{B}_{\mu}^{a}(x)=\frac{g^{2}}{2} \int_{\beta} d^{2} y b_{(\mu)}^{a b}(y, x) \bar{B}_{\mu}^{b}(y),
$$

with $b_{(\mu)}^{a b}(y, x)$ satisfying

$$
\int d^{2} y b_{(\mu)}^{a b}(y, x) V_{(\mu)}^{b c}(z, y)=\delta^{a c} \delta^{2}(x-z) .
$$

At this point we make the change

$$
\begin{aligned}
& \frac{g}{2}\left(\tilde{A}_{\mu}^{a}+\bar{B}_{\mu}^{a}\right)=A_{\mu}^{a}, \\
& \frac{g}{2}\left(\tilde{A}_{\mu}^{a}-\bar{B}_{\mu}^{a}\right)=B_{\mu}^{a},
\end{aligned}
$$

which allows us to write 


$$
\begin{aligned}
Z= & N_{1} N_{F}(\beta) \int_{\text {periodic }} D A_{\mu}^{a} D B_{\mu}^{b} \operatorname{det}_{\beta}(i \not \partial-g A) \\
& \times \exp \left\{\frac{-1}{2} \int_{\beta} d^{2} x d^{2} y\left[b_{(\mu)}^{a b}(y, x) A_{\mu}^{a}(x) A_{\mu}^{b}(y)-b_{(\mu)}^{a b}(x, y) B_{\mu}^{a}(x) B_{\mu}^{b}(y)\right]\right\},
\end{aligned}
$$

where we have used the fact that the functions $V_{(\mu)}^{a b}$ and $b_{(\mu)}^{a b}$ are symmetric in coordinates. Note that the Jacobian associated with the change $(\tilde{A}, \tilde{B}) \rightarrow(A, B)$ does not depend either on fields or on temperature and then it can be absorbed in the normalization constant $\left(N \rightarrow N_{1}\right)$.

We have been able to express $Z$ in terms of a temperature-dependent fermionic determinant. This fact will enable us to apply the non-local bosonization scheme, first developed for $T=0[4]$, to the present finite-temperature case.

Note that, as a consequence of the change of bosonic variables (Eqs. (2.18) and (2.19)), the effect of the non-local interaction has been completely transferred to the purely bosonic piece of the action, $S[A, B]$. On the other hand, we see that the field $B_{\mu}$ is completely decoupled from both the $A_{\mu}$-field and the fermion field. This clearly indicates that its contribution should be factorized and absorbed in the normalization constant. However, the issue is more subtle since for repulsive interactions $B_{\mu}$ corresponds to a negative-metric state. This is not a peculiar feature of neither non-local nor finite-temperature theories. Indeed, the appearance of negative-metric states was already stressed by Klaiber in his seminal work on the usual Thirring model [8]. In order to have a well-defined Hilbert space, Klaiber had to disregard these fields. Following the same prescription in this new context, we are naturally led to include the decoupled $B_{\mu}$-integral in $N_{F}(\beta)$. Indeed, as it is habitual in finite-temperature studies, $N_{F}(\beta)$ contains all the $\beta$-dependent infinite contributions to the partition function. Since the integral over ghost fields is also a $\beta$-dependent infinite factor (otherwise one would not reproduce the well-known result for the $T \neq 0$ local Thirring model (see Eq. (2.62))), it is natural to include it in $N_{F}(\beta)$. The partition function $(2.20)$ then reads

$$
Z=N_{F}(\beta) N_{1} \int_{\text {periodic }} D A_{\mu}^{a} \operatorname{det}(i \not-g A) e^{-S\left\{A_{\mu}\right\rfloor},
$$

where $S[A]$ is the $A_{\mu}$-dependent part of (2.20). From now on we shall take the fermion fields in the fundamental representation of the maximal abelian subgroup of $U(2)$. In the many-body language this corresponds to a system of spin- $\frac{1}{2}$ fermions in which spin-flipping processes are forbidden.

Now, the potential matrices are diagonal whose elements can be written in terms of Sólyom's "g-ology" [9] as

$$
\begin{aligned}
& V_{(0)}^{00}=\frac{1}{4}\left(g_{4 \|}+g_{4 \perp}+g_{2 \|}+g_{2 \perp}\right), \\
& V_{(0)}^{11}=\frac{1}{4}\left(g_{4 \|}-g_{4 \perp}+g_{2 \|}-g_{2 \perp}\right),
\end{aligned}
$$




$$
\begin{aligned}
& V_{(I)}^{00}=\frac{1}{4}\left(-g_{4 \|}-g_{4 \perp}+g_{2 \|}+g_{2 \perp}\right), \\
& V_{(1)}^{11}=\frac{1}{4}\left(-g_{4 \|}+g_{4 \perp}+g_{2 \|}-g_{2 \perp}\right),
\end{aligned}
$$

where $g_{2}$ and $g_{4}$ are associated to scattering diagrams involving two or just one electronic species (left- or right-moving), respectively. On the other hand, the subscripts $\|$ and $\perp$ denote those processes in which incident fermions have parallel or anti-parallel spins. Let us also recall for later convenience that the Tomonaga-Luttinger model, in which only charge-density fluctuations are considered, corresponds to $V_{(1)}^{00}=V_{(1)}^{11}=0$.

At this stage we can decouple the vector field $A_{\mu}$ from the fermion fields in the fermionic determinant contained in (2.21). The only new feature in this otherwise standard step, is given by the modified $\gamma_{1}$ matrix of Eq. (2.3). Fortunately this fact does not pose any substantial problem. Indeed, writing the components of $A_{\mu}$ in terms of two scalar fields $\phi$ and $\eta$ in the form

$$
\begin{aligned}
& A_{0}(x)=v_{F} \partial_{1} \phi(x)-\partial_{0} \eta(x), \\
& A_{1}(x)=\frac{-1}{v_{F}} \partial_{0} \phi(x)-\partial_{1} \eta(x)
\end{aligned}
$$

with $\phi=\phi^{i} \lambda^{i}, \eta=\eta^{i} \lambda^{i}, i=0,1$, it is easy to verify that the desired decoupling is achieved by the same chiral transformation in the fermionic variables that one would perform in the $v_{F}=1$ case:

$$
\begin{aligned}
& \Psi(x)=e^{g\left[\gamma_{5} \phi(x)+i \eta(x)\right]} \chi(x), \\
& \bar{\Psi}(x)=\bar{\chi}(x) e^{g\left[\gamma_{5} \phi(x)-i \eta(x)\right]},
\end{aligned}
$$

where $\gamma_{5}$ is the usual chiral matrix, i.e. the one that is obtained in the case $v_{F}=1$. Thus we get

$$
\operatorname{det}_{\beta}(i \not \partial-g \not \hat{A})=J_{F}[\phi, \eta] \operatorname{det}_{\beta} i \not
$$

and the Jacobian of the fermionic transformation is given by [10]

$$
\ln J_{F}[\phi, \eta]=\frac{-g^{2}}{2 \pi} \int_{\beta} d^{2} x\left[\frac{1}{v_{F}}\left(\partial_{0} \phi\right)^{2}+v_{F}\left(\partial_{l} \phi\right)^{2}\right] .
$$

Concerning the bosonic change of variables given by (2.23) and (2.24), one has to consider another temperature-dependent Jacobian, to be included in the path-integral measure as

$$
D A_{\mu}=\operatorname{det}_{\beta}(-\square) D \phi^{a} D \eta^{a},
$$

where $\square=\frac{1}{v_{F}} \partial_{0}^{2}+v_{F} \partial_{1}^{2}$.

Inserting (2.27), (2.28), and (2.29) in (2.21) the result is

$$
Z=N_{1} N_{F}(\beta) \operatorname{det}_{\beta}^{2}(i \not \partial) \operatorname{det}_{\beta}^{2}(-\square) \int \prod_{a=0,1} D \phi^{a} D \eta^{a} \exp \left\{-S_{\mathrm{eff}}^{a a}[\phi, \eta]\right\},
$$

where 


$$
\begin{aligned}
S_{\mathrm{eff}}^{a a}[\phi, \eta]= & \frac{\lambda}{2} \int_{\beta} d^{2} x\left[\frac{1}{v_{F}}\left(\partial_{0} \phi^{a}\right)^{2}+v_{F}\left(\partial_{1} \phi^{a}\right)^{2}\right] \\
& +\frac{1}{2} \int_{\beta} d^{2} x d^{2} y\left[v_{F}^{2} \partial_{1} \phi^{a}(x) b_{(0)}^{a a}(x, y) \partial_{1} \phi^{a}(y)\right. \\
& +\frac{1}{v_{F}^{2}} \partial_{0} \phi^{a}(x) b_{(1)}^{a a}(x, y) \partial_{0} \phi^{a}(y)+\partial_{0} \eta^{a} b_{(0)}^{a a}(x, y) \partial_{0} \eta^{a}(y) \\
& +\partial_{1} \eta^{a}(x) b_{(1)}^{a a}(x, y) \partial_{1} \eta^{a}(y)+2 v_{F} \partial_{0} \eta^{a}(x) b_{(0)}^{a a}(x, y) \partial_{1} \phi^{a}(y) \\
& \left.-\frac{2}{v_{F}} \partial_{1} \eta^{a}(x) b_{(1)}^{a a}(x, y) \partial_{0} \phi^{a}(y)\right]
\end{aligned}
$$

and $\lambda=g^{2} / \pi$.

In order to evaluate $\ln Z$ we follow the pioneering work of Bernard [5] and expand the bosonic fields $\phi\left(x^{0}, x^{1}\right)$ and $\eta\left(x^{0}, x^{1}\right)$, which are periodic in the interval $0 \leqslant x \leqslant \beta$, in Fourier series,

$$
\begin{aligned}
& \phi^{a}\left(x^{0}, x^{1}\right)=\frac{1}{\beta} \sum_{n=-\infty}^{\infty} \int \frac{d k_{1}}{2 \pi} e^{i k_{1} x^{1}} e^{i \omega_{n} x^{0}} \tilde{\phi}_{n}^{a}\left(k_{1}\right), \\
& \eta^{a}\left(x^{0}, x^{1}\right)=\frac{1}{\beta} \sum_{n=-\infty}^{\infty} \int \frac{d k_{1}}{2 \pi} e^{i k_{1} x^{1}} e^{i \omega_{n} x^{0}} \tilde{\eta}_{n}^{a}\left(k_{1}\right),
\end{aligned}
$$

where

$$
\begin{aligned}
\tilde{\phi}_{n}^{a}\left(k_{1}\right) & =\int d x^{1} \int_{0}^{\beta} d x^{0} e^{-i k_{1} x^{1}} e^{-i \omega_{n} x^{0}} \phi^{a}\left(x^{0}, x^{1}\right), \\
\tilde{\eta_{n}^{a}}\left(k_{1}\right) & =\int d x^{1} \int_{0}^{\beta} d x^{0} e^{-i k_{1} x^{1}} e^{-i \omega_{n} x^{0}} \eta^{a}\left(x^{0}, x^{1}\right)
\end{aligned}
$$

and the Matsubara frequencies are given by

$$
\omega_{n}=\frac{2 n \pi}{\beta} .
$$

In an analogous way one can expand the inverse potentials as

$$
b_{(\mu)}^{a b}\left(x^{1}, y^{1}, x^{0}, y^{0}\right)=\frac{1}{\beta} \sum_{n=-\infty}^{\infty} \int \frac{d k_{1}^{\prime}}{2 \pi} e^{i k_{1}^{\prime}\left(x^{1}-y^{1}\right)} e^{i \omega_{n^{\prime}}\left(x^{0}-y^{0}\right)} \tilde{b}_{(\mu) n^{\prime}}^{a b}\left(k_{1}^{\prime}\right) .
$$

In writing the above expressions we have used discrete and continuous delta functions defined by

$$
\int_{0}^{\beta} d x^{0} e^{i\left(\omega_{n}-\omega_{n^{\prime}}\right) x^{0}}=\beta \delta_{n, n^{\prime}}
$$




$$
\int \frac{d x^{1}}{2 \pi} e^{i\left(k_{1}-k_{1}^{\prime}\right) x^{1}}=\delta\left(k_{1}-k_{1}^{\prime}\right)
$$

Eq. (2.31) can then be rewritten as

$$
\begin{aligned}
S_{\mathrm{eff}}^{a a}= & \frac{1}{2 \beta} \sum_{n=-\infty}^{\infty} \int \frac{d k_{1}}{2 \pi}\left[\tilde{\phi}_{n}^{a}\left(k_{1}\right) \tilde{\phi}_{-n}^{a}\left(-k_{1}\right) A_{n}^{a a}\left(k_{1}\right)\right. \\
& \left.+\tilde{\eta}_{n}^{a}\left(k_{1}\right) \tilde{\eta}_{-n}^{a}\left(-k_{1}\right) B_{n}^{a a}\left(k_{1}\right)+\tilde{\phi}_{n}^{a}\left(k_{1}\right) \tilde{\eta}_{-n}^{a}\left(-k_{1}\right) C_{n}^{a a}\left(k_{1}\right)\right],
\end{aligned}
$$

where

$$
\begin{aligned}
& A_{n}^{a a}\left(k_{1}\right)=\lambda\left(\frac{\omega_{n}^{2}}{v_{F}}+v_{F} k_{1}^{2}\right)+v_{F}^{2} k_{1}^{2} \tilde{b}_{(0), n}^{a a}\left(k_{1}\right)+\tilde{b}_{(1), n}^{a a}\left(k_{1}\right) \frac{\omega_{n}^{2}}{v_{F}^{2}}, \\
& B_{n}^{a a}\left(k_{1}\right)=\tilde{b}_{(0), n}^{a a}\left(k_{1}\right) \omega_{n}^{2}+\tilde{b}_{(1), n}^{a a}\left(k_{1}\right) k_{1}^{2}, \\
& C_{n}^{a a}\left(k_{1}\right)=2\left[v_{F} \tilde{b}_{(0), n}^{a a}\left(k_{1}\right)-\frac{1}{v_{F}} \tilde{b}_{(1), n}^{a a}\left(k_{1}\right)\right] \omega_{n} k_{1} .
\end{aligned}
$$

The action (2.40) can be easily diagonalized through the change

$$
\begin{aligned}
\phi_{n}^{a} & =\zeta_{n}^{a}-\frac{C_{-n}^{a a}}{2 A_{-n}^{a a}} \xi_{n}^{a}, \\
\eta_{n}^{a} & =\xi_{n}^{a},
\end{aligned}
$$

which yields

$$
S_{\mathrm{eff}}^{a a}=\frac{1}{2 \beta} \sum_{n=-\infty}^{\infty} \int \frac{d k_{1}}{2 \pi}\left[\zeta_{n}^{a}\left(k_{1}\right) G_{\zeta, n}^{a a}\left(k_{1}\right) \zeta_{-n}^{a}\left(-k_{1}\right)+\xi_{n}^{a}\left(k_{1}\right) G_{\xi, n}^{a a}\left(k_{1}\right) \xi_{-n}^{a}\left(-k_{1}\right)\right],
$$

where

$$
G_{\zeta, n}^{a a}\left(k_{1}\right)=\lambda\left(v_{F} k_{1}^{2}+\frac{\omega_{n}^{2}}{v_{F}}\right)+\left[\tilde{b}_{(0)}^{a a} v_{F}^{2} k_{1}^{2}+\tilde{b}_{(1)}^{a a} \frac{\omega_{n}^{2}}{v_{F}^{2}}\right]
$$

and

$$
G_{\xi, n}^{a a}\left(k_{1}\right)=\frac{\lambda\left(\omega_{n}^{2} / v_{F}+v_{F} k_{1}^{2}\right)\left[\tilde{b}_{(0), n}^{a a} \omega_{n}^{2}+\tilde{b}_{(1), n}^{a a} k_{1}^{2}\right]+\tilde{b}_{(0), n}^{a a} \tilde{b}_{(1), n}^{a a}\left(v_{F} k_{1}^{2}+\omega_{n}^{2} / v_{F}\right)^{2}}{\lambda\left(v_{F} k_{1}^{2}+\omega_{n}^{2} / v_{F}\right)+\tilde{b}_{(0), n}^{a a} v_{F}^{2} k_{1}^{2}+\tilde{b}_{(1), n}^{a a} \omega_{n}^{2} / v_{F}} .
$$

The partition function of the system can now be expressed as

$$
\left.Z=N_{1} N_{F}(\beta) \operatorname{det}_{\beta}^{2}(-\square) \operatorname{det}_{\beta}^{2}(i \not)\right) \prod_{a=0,1}\left(\operatorname{det} G_{\zeta, n}^{a a}\right)^{-1 / 2}\left(\operatorname{det} G_{\xi, n}^{a a}\right)^{-1 / 2}
$$

and its logarithm, using the well-known identity involving functional traces and determinants, can be written as 


$$
\ln Z=\ln Z^{00}+\ln Z^{11}
$$

where we have defined

$$
\ln Z^{a a}=\frac{1}{2} \ln N_{F}(\beta)+\frac{1}{2} \ln N_{1}+\operatorname{tr} \ln (i \phi)+\operatorname{tr} \ln (-\square)-\frac{1}{2} \operatorname{tr} \ln G_{\zeta, n}^{a a}-\frac{1}{2} \operatorname{tr} \ln G_{\xi, n}^{a a} .
$$

Since in general one is interested in derivatives of the partition functions with respect to temperature, we shall disregard $\beta$-independent terms. In particular, from now on any reference to $N_{1}$ will be omitted.

The last two terms in (2.51) can be expressed in the form

$$
\begin{aligned}
-\frac{1}{2} \operatorname{tr} \ln G_{\zeta, n}^{a a}-\frac{1}{2} \operatorname{tr} \ln G_{\xi, n}^{a a}= & -\frac{1}{2} \operatorname{tr} \ln \left[\tilde{b}_{(0), n}^{a a}\left(k_{1}\right)\left(\lambda+\frac{\tilde{b}_{(1), n}^{a a}\left(k_{1}\right)}{v_{F}}\right)\right] \\
& -\frac{1}{2} \operatorname{tr} \ln \left[v_{F} k_{1}^{2}+\frac{\omega_{n}^{2}}{v_{F}}\right]-\frac{1}{2} \operatorname{tr} \ln \left[\omega_{n}^{2}+k_{1}^{2} f_{n}^{a a}\left(k_{1}\right)\right]
\end{aligned}
$$

with

$$
f_{n}^{a a}\left(k_{1}\right)=\frac{\tilde{b}_{(1), n}^{a a}}{\tilde{b}_{(0), n}^{a a}} \frac{\lambda+v_{F} \tilde{b}_{(0), n}^{a a}}{\lambda+\tilde{b}_{(1), n}^{a a} / v_{F}} .
$$

Using also the well-known identity [5]

$$
\begin{aligned}
\operatorname{tr} \ln \left(v_{F} k_{1}^{2}+\frac{1}{v_{F}} \omega_{n}^{2}\right) & =\sum_{n=-\infty}^{\infty} \int \frac{d k_{1}}{2 \pi} \ln \left(\frac{1}{v_{F}} \omega_{n}^{2}+v_{F} k_{1}^{2}\right) \\
& =\operatorname{tr} \ln (-\square)=\ln \operatorname{det}_{\beta}(-\square)
\end{aligned}
$$

and replacing (2.52) and (2.54) in (2.51) one obtains

$$
\begin{aligned}
\ln Z^{a a}= & \frac{1}{2} \ln N_{F}(\beta)+\ln \operatorname{det}_{\beta} i \not \partial+\frac{1}{2} \ln \operatorname{det}_{\beta}(-\square) \\
& -\frac{1}{2} \operatorname{tr} \ln \left[\tilde{b}_{(0), n}^{a a}\left(k_{1}\right)\left(\lambda+\frac{\tilde{b}_{1}^{a a}}{v_{F}}\right)\right]-\frac{1}{2} \operatorname{tr} \ln \left[\omega_{n}^{2}+k_{1}^{2} f_{n}^{a a}\left(k_{1}\right)\right] .
\end{aligned}
$$

Let us recall that up to now we have considered a very general situation. Indeed, the above formula is valid for potentials that depend on both distances and the temperature. However, from a physical point of view it is reasonable to assume that the interactions are temperature-independent. In this case we can generalize the procedure developed in Refs. $[5,11]$ in order to write

$$
\begin{aligned}
\frac{-1}{2} \operatorname{tr} & \ln \left[\omega_{n}^{2}+k_{1}^{2} f^{a a}\left(k_{1}\right)\right]=\frac{1}{2} \int \frac{d k_{1}}{2 \pi}\left\{(1-k \beta)\left(f^{a a}\right)^{1 / 2}(k)\right. \\
& \left.-2 \ln \left(1-e^{-k \beta\left(f^{a a}\right)^{1 / 2}(k)}\right)+2 \ln \left(1-e^{-\left(f^{a a}\right)^{1 / 2}(k)}\right)\right\} \\
& -\frac{1}{2} \int \frac{d k_{1}}{2 \pi} \sum_{n=-\infty}^{\infty} \ln \left[(2 \pi n)^{2}+f^{a a}(k)\right]+\ln \beta \int \frac{d k_{1}}{2 \pi} \sum_{n=-\infty}^{\infty},
\end{aligned}
$$




$$
\begin{aligned}
& \frac{1}{2} \ln \operatorname{det}_{\beta}(-\square)=\int \frac{d k_{1}}{2 \pi} \frac{v_{F} k \beta}{2}+\int \frac{d k_{1}}{2 \pi} \ln \left(1-e^{-v_{F} k \beta}\right)-\int \frac{d k_{1}}{2 \pi} \ln \beta \sum_{n=-\infty}^{\infty} \\
& \frac{1}{2} \ln \operatorname{det}_{\beta}(i \phi)=\int \frac{d k_{1}}{2 \pi} \frac{v_{F} k \beta}{2}+\int \frac{d k_{1}}{2 \pi} \ln \left(1+e^{-v_{F} k \beta}\right)-\int \frac{d k_{1}}{2 \pi} \ln \beta \sum_{n=-\infty}^{\infty},
\end{aligned}
$$

where $k=\left|k_{1}\right|$. Note that here we are calling $f^{a a}$ the expression (2.53) in the present, $n$-independent case. As usual, the $\beta$-dependent infinite contributions to the partition function are eliminated by choosing

$$
\ln N_{F}(\beta)=2 \ln \beta \sum_{n=-\infty}^{\infty} \int \frac{d k_{1}}{2 \pi}
$$

Thus we finally obtain

$$
\begin{aligned}
\ln Z^{a a}= & \int \frac{d k_{1}}{2 \pi}\left\{\frac{3}{2} v_{F} k \beta+2 \ln \left(1+e^{-v_{F} k \beta}\right)+\ln \left(1-e^{-v_{F} k \beta}\right)\right\} \\
& +\frac{1}{2} \int \frac{d k_{1}}{2 \pi}\left[(1-k \beta)\left(f^{a a}\right)^{1 / 2}(k)-2 \ln \left(1-e^{-k \beta\left(f^{a a}\right)^{1 / 2}(k)}\right)\right. \\
& \left.+2 \ln \left(1-e^{-\left(f^{a a}\right)^{1 / 2}(k)}\right)\right]-\frac{1}{2} \int \frac{d k_{1}}{2 \pi} \sum_{n=-\infty}^{\infty} \ln \left[(2 \pi n)^{2}+f^{a a}(k)\right] \\
& -\frac{1}{2} \int \frac{d k_{1}}{2 \pi} \ln \left[\tilde{b}_{(0)}^{a a}(k)\left(\lambda+\frac{\tilde{b}_{(1)}^{a a}(k)}{v_{F}}\right)\right] \sum_{n=-\infty}^{\infty},
\end{aligned}
$$

which is our first non-trivial result. Indeed, by inserting this expression in (2.50) we have the partition function corresponding to the Thirring model with non-local interactions. Therefore, it is the extension of the well-known results for the local Thirring model at finite temperature (see Refs. [12,7] and references therein) to the case in which non-contact potentials are taken into account. Apart from academic interest, this result could be useful in order to explore thermodynamical properties in strongly correlated systems, since for $b_{(1)}^{a a}=\infty$ the NLT model describes a TL system [4]. Note that, in the case $b_{(0)}^{a a}=b_{(1)}^{a a}=b^{a a}(x, y)$ and $v_{F}=1$ (which leads to $f^{a a}(k)=1$ ), one obtains a simplified non-local covariant model:

$$
\ln Z^{a a}=2 \int \frac{d k_{1}}{2 \pi}\left\{\frac{k \beta}{2}+\ln \left(1+e^{-k \beta}\right)\right\}-\frac{1}{2} \int \frac{d k_{1}}{2 \pi} \sum_{n=-\infty}^{\infty} \ln \left[\tilde{b}^{a a}(k)\left(\lambda+\tilde{b}^{a a}(k)\right)\right]
$$

which for $b_{0}^{a a}=b_{(1)}^{a a}=\delta^{2}(x-y)$ gives the right result for the usual (local) Thirring model at finite temperature $[12,7]$ :

$$
\ln Z_{\text {Thirring }}=2 \int \frac{d k_{1}}{2 \pi}\left\{\frac{k \beta}{2}+\ln \left(1+e^{-k \beta}\right)-\frac{1}{2} \ln (\lambda+1) \sum_{n=-\infty}^{\infty}\right\} .
$$




\section{Thermodynamical functions}

In the previous section we evaluated the partition function for the non-local Thirring model. The main purpose of the present section is to derive analytical expressions for relevant thermodynamical functions such as the Helmholtz free energy, the energy and the specific heat. Throughout this section we shall consider $\beta$-independent potentials. Let us begin with the Helmholtz free energy defined as

$$
F=-\frac{1}{\beta} \ln Z=F^{00}+F^{11},
$$

where

$$
\begin{aligned}
F^{a a}= & -\int \frac{d k_{1}}{2 \pi}\left\{\frac{3 k v_{F}}{2}+\frac{2}{\beta} \ln \left(1+e^{-k \beta v_{F}}\right)+\frac{1}{\beta} \ln \left(1-e^{-k \beta v_{F}}\right)\right. \\
& -\frac{1}{\beta} \ln \left(1-e^{-k \beta\left(f^{(a a)}\right)^{1 / 2}(k)}\right)+\frac{\left(f^{a a}\right)^{1 / 2}(k)}{2 \beta}(1-k \beta) \\
& +\frac{1}{\beta} \ln \left(1-e^{-\left(f^{a a}\right)^{1 / 2}(k)}\right)-\frac{1}{2 \beta} \sum_{n} \ln \left[(2 \pi n)^{2}+f^{a a}(k)\right] \\
& \left.-\frac{1}{2 \beta} \ln \left[\tilde{b}_{(0)}^{a a}(k)\left(\lambda+\tilde{b}_{(1)}^{a a}(k)\right)\right] \sum_{n=-\infty}^{\infty}\right\} .
\end{aligned}
$$

The energy is given by $E=-\partial \ln Z / \partial \beta$. Using Eq. (2.60), ignoring the zero-point energy of the vacuum and taking into account that

$$
2 \int_{0}^{\infty} \frac{d k}{2 \pi} \frac{k v_{F}}{e^{k \beta v_{F}}+1}=\int_{0}^{\infty} \frac{d k}{2 \pi} \frac{k v_{F}}{e^{k \beta v_{F}}-1}=\frac{\pi}{12 \beta^{2} v_{F}}
$$

we obtain

$$
\begin{aligned}
E=E^{00}+E^{11}= & \int \frac{d k_{1}}{2 \pi}\left\{\frac{\left(f^{00}\right)^{1 / 2}(k)+\left(f^{11}\right)^{1 / 2}}{2}\right. \\
& \left.+\frac{k\left(f^{00}\right)^{1 / 2}(k)}{e^{k \beta\left(f^{00}\right)^{1 / 2}}-1}+\frac{k\left(f^{11}\right)^{1 / 2}(k)}{e^{k \beta\left(f^{11}\right)^{1 / 2}}-1}\right\},
\end{aligned}
$$

where, since we have supposed that the electron-electron potentials are $\beta$-independent, one has $f^{a a}(k, \omega)=f^{a a}(k)$.

Performing the derivative of the above expression with respect to temperature, one can write the specific heat as

$$
\begin{aligned}
C_{V}= & \frac{1}{k_{\mathrm{B}} T^{2}} \int \frac{d k_{1}}{2 \pi} k^{2}\left\{f^{00}(k) \frac{e^{k\left(f^{00}\right)^{1 / 2}(k) / k_{\mathrm{B}} T}}{\left(1-e^{k\left(f^{00}\right)^{1 / 2}(k) / k_{\mathrm{B}} T}\right)^{2}}\right. \\
& \left.+f^{11}(k) \frac{e^{k\left(f^{11}\right)^{1 / 2}(k) / k_{\mathrm{B}} T}}{\left(1-e^{k\left(f^{11}\right)^{1 / 2}(k) / k_{\mathrm{B}} T}\right)^{2}}\right\} .
\end{aligned}
$$


Recalling the dispersion relations for charge-density and spin-density modes given in [4], one can easily write $f^{00}$ and $f^{11}$ in terms of the corresponding velocities $v_{\rho}$ and $v_{\sigma}$ as

$$
\begin{aligned}
& f^{00}=v_{F}^{2} v_{\rho}^{2}, \\
& f^{11}=v_{F}^{2} v_{\sigma}^{2}
\end{aligned}
$$

In order to check our general formula (3.6) we can consider the local case, in which $v_{\rho}$ and $v_{\sigma}$ are constants. For this particular case we can easily compute the corresponding integral, which yields

$$
C_{V}=k_{\mathrm{B}}^{2} \frac{\pi}{3}\left(\frac{1}{v_{\rho}}+\frac{1}{v_{\sigma}}\right) T,
$$

which is the characteristic linear behavior of the underlying fermions (linear specific heat in any dimension) when only weak interactions (consistent with keeping only forward-scattering interactions) are taken into account. Thus, in this section we have presented analytical expressions for the energy and the specific heat of a general forwardscattering interacting system of fermions, represented by the non-local Thirring model. Our formulae give the thermodynamical magnitudes not only as functions of temperature, but as functionals of the electron-electron potentials. Then, they could be used in order to examine the influence of different potentials on the equilibrium properties of the $1 \mathrm{~d}$ electron liquid.

\section{Two-point fermionic correlations}

Let us now go back to the general case, i.e. with potentials not necessarily $\beta$ independent, and compute the fermionic propagator defined by

$$
\left\langle\Psi(\tau, x) \vec{\Psi}\left(\tau^{\prime}, y\right)\right\rangle_{\beta}=\left(\begin{array}{cc}
0 & G_{+}^{\beta}\left(\tau, x, \tau^{\prime}, y\right) \\
G_{-}^{\beta}\left(\tau, x, \tau^{\prime}, y\right) & 0
\end{array}\right),
$$

where

$$
G_{( \pm)}^{\beta}\left(\tau, x, \tau^{\prime}, y\right)=\left(\begin{array}{cc}
G_{( \pm) \uparrow}^{\beta}\left(\tau, x, \tau^{\prime}, y\right) & 0 \\
0 & G_{( \pm) \downarrow}^{\beta}\left(\tau, x, \tau^{\prime}, y\right)
\end{array}\right) .
$$

The subindex $+(-)$ means that we consider right(left)-moving electrons, and $\uparrow(\downarrow)$ indicates that the field operator carries a spin up (down) quantum number. In the present case we have disregarded those processes with spin-flip. To be specific we shall restrict our analysis to $G_{( \pm) \uparrow}^{\beta}$ (similar expressions are obtained for $G_{( \pm) \downarrow}^{\beta}$ ).

Once we have performed the decoupling change of variables given by Eqs. (2.25) and (2.26) the non-vanishing components of the thermal Green function are factorized in the form 


$$
\begin{aligned}
G_{ \pm \uparrow}^{\beta}\left(x, \tau, y, \tau^{\prime}\right)=\left\langle\Psi_{ \pm \uparrow}(x) \bar{\Psi}_{ \pm \uparrow}(y)\right\rangle_{\beta}= & G_{ \pm \uparrow \beta}^{(0)}\left(x, \tau, y, \tau^{\prime}\right) B_{ \pm \beta}^{00}\left(x, \tau, y, \tau^{\prime}\right) \\
& \times B_{ \pm \beta}^{11}\left(x, \tau, y, \tau^{\prime}\right),
\end{aligned}
$$

where $G_{ \pm \uparrow \beta}^{(0)}\left(x, \tau, y, \tau^{\prime}\right)$ is the free thermal Green function given by

$$
G_{ \pm \uparrow \beta}^{(0)}\left(\tau-\tau^{\prime}, x-y\right)=e^{ \pm i u_{F} p_{F}(x-y)} \sum_{n=-\infty}^{\infty} \frac{1}{2 \pi \beta} \int d k_{1} \frac{e^{-i\left(\omega_{n}\left(\tau-\tau^{\prime}\right)+k_{1}(x-y)\right)}}{\omega_{n} \mp i k_{1} v_{F}}
$$

with $\omega_{n}=(2 n+1) \pi / \beta$, and

$$
\begin{aligned}
& B_{ \pm \beta}^{00}=\left\langle e^{g\left[\phi^{0}\left(y, \tau^{\prime}\right)-\phi^{0}(x, \tau)\right]} e^{i g\left[\eta^{0}\left(y, \tau^{\prime}\right)-\eta^{0}(x, \tau)\right]}\right\rangle_{ \pm \beta}^{00}, \\
& B_{ \pm \beta}^{11}=\left\langle e^{g\left[\phi^{1}\left(y, \tau^{\prime}\right)-\phi^{1}(x, \tau)\right]} e^{i g\left[\eta^{1}\left(y, \tau^{\prime}\right)-\eta^{\prime}(x, \tau)\right]}\right\rangle_{ \pm \beta}^{11} .
\end{aligned}
$$

In Eq. (4.4) we have redefined the energy origin, as usual, by introducing the Fermi momentum $p_{F}$. In Eqs. (4.5) and (4.6) the symbol \langle\rangle$_{\beta}^{a a}$ means v.e.v. with respect to the action (2.31). Working in momentum space these bosonic factors can be written as

$$
B_{ \pm \beta}^{a a}\left(x, \tau, y, \tau^{\prime}\right)=\frac{\int D \tilde{\phi}^{a} D \tilde{\eta}^{a} e^{-\left[S_{\mathrm{eff}}^{a a}+S_{ \pm \beta}^{a a}\left(x, \tau, y, \tau^{\prime}\right)\right]}}{\int D \tilde{\phi}^{a} D \tilde{\eta}^{a} e^{-S_{\mathrm{eff}}^{a a}}}
$$

with $S_{\text {eff }}$ given by Eq. (2.40) and

$$
\begin{aligned}
S_{ \pm \beta}^{a a}\left(x, \tau, y, \tau^{\prime}\right) & =\frac{g}{\beta} \sum_{n} \int \frac{d k_{1}}{2 \pi}\left[ \pm \tilde{\phi}_{n}^{a}\left(k_{1}\right)+i \tilde{\eta}_{n}^{a}\left(k_{1}\right)\right] D\left(\omega_{n}, k_{1} ; x, \tau, y, \tau^{\prime}\right), \\
D\left(\omega_{n}, k_{1} ; x, \tau, y, \tau^{\prime}\right) & =e^{i \omega_{n} \tau} e^{i k_{1} \cdot x}-e^{i \omega_{n}\left(\tau^{\prime}\right)} e^{i k_{1} \cdot y}
\end{aligned}
$$

Now $B_{ \pm \beta}^{a a}$ can be easily evaluated by performing the change

$$
\begin{aligned}
& \tilde{\phi}_{n}^{a}\left(k_{1}\right)=\tilde{\varphi}_{n}^{a}\left(k_{1}\right)+E_{n, \pm}^{a a}\left(k_{1} ; x, \tau, y, \tau^{\prime}\right), \\
& \tilde{\eta}_{n}^{a}\left(k_{1}\right)=\tilde{\rho}_{n}^{a}\left(k_{1}\right)+F_{n, \pm}^{a a}\left(k_{1} ; x, \tau, y, \tau^{\prime}\right),
\end{aligned}
$$

where $\tilde{\varphi}^{a}$ and $\tilde{\rho}^{a}$ are the new quantum variables and $E_{n, \pm}^{a a}$ and $F_{n, \pm}^{a a}$ are classical functions chosen in the form

$$
\begin{aligned}
E_{n, \pm}^{a a}\left(k_{1} ; x, \tau, y, \tau^{\prime}\right) & =g\left[\frac{i C_{n}^{a a}\left(k_{1}\right)}{2} \mp B_{n}^{a a}\left(k_{1}\right)\right] \frac{D\left(\omega_{n}, k_{1} ; x, \tau, y, \tau^{\prime}\right)}{\Delta_{n}^{a a}\left(k_{1}\right)} \\
F_{n, \pm}^{a a}\left(k_{1}\right) & =g\left[ \pm \frac{C^{a a}\left(k_{1}\right)}{2}-i A_{n}^{a a}\left(k_{1}\right)\right] \frac{D\left(\omega_{n}, k_{1} ; x, y\right)}{\Delta_{n}^{a a}\left(k_{1}\right)}
\end{aligned}
$$

with $A_{n}^{a a}\left(k_{1}\right), B_{n}^{a a}\left(k_{1}\right)$ and $C_{n}^{a a}\left(k_{1}\right)$ defined in $((2.41)-(2.43))$ and

$$
\Delta_{n}^{a a}\left(k_{1}\right)=\left(C_{n}^{a a}\right)^{2}\left(k_{1}\right)-4 A_{n}^{a a}\left(k_{1}\right) B_{n}^{a a}\left(k_{1}\right) .
$$

Putting all this together, the result is 


$$
\begin{aligned}
B_{ \pm \beta}^{a a}\left(x, \tau, y, \tau^{\prime}\right)= & \exp \left\{-\frac{2 g^{2}}{\beta} \sum_{n=-\infty}^{\infty} \int \frac{d k_{1}}{2 \pi} D\left(\omega_{n}, k_{1} ; x, \tau, y, \tau^{\prime}\right)\right. \\
& \left.\times D\left(\omega_{-n},-k_{1} ; x, \tau, y, \tau^{\prime}\right) \frac{B_{n}^{a a}\left(k_{1}\right)-A_{n}^{a a}\left(k_{1}\right) \mp i C_{n}^{a a}\left(k_{1}\right)}{\Delta_{n}^{a a}\left(k_{1}\right)}\right\}
\end{aligned}
$$

which can also be written as

$$
\begin{aligned}
B_{ \pm \beta}^{a a}\left(x, \tau, y, \tau^{\prime}\right)= & \exp \left\{-\frac{8 g^{2}}{\beta} \sum_{n=-\infty}^{\infty} \int \frac{d k_{1}}{2 \pi} \operatorname{sen}^{2}\left[\frac{\omega_{n}\left(\tau-\tau^{\prime}\right)}{2}+\frac{k_{1}(x-y)}{2}\right]\right. \\
& \left.\times \frac{B_{n}^{a a}\left(k_{1}\right)-A_{n}^{a a}\left(k_{1}\right) \mp i C_{n}^{a a}\left(k_{1}\right)}{\Delta_{n}^{a a}\left(k_{1}\right)}\right\} .
\end{aligned}
$$

This is the main result of this section. Indeed, apart from the free fermion contribution given by Eq. (4.4), Eq. (4.15) gives the fermionic thermal propagator as functional of the electron-electron potentials. Please note that up to this point these couplings are allowed to depend on both distance and temperature. Of course, in order to go further in this computation one needs to specify the Fourier transformed inverse potentials $\tilde{b}_{(\mu), n}\left(k_{1}\right)$ which determine the integrand in (4.15). The simplest case at hand is the one corresponding to the usual Thirring model, which once again can be used to check the consistency of our more general calculation. Setting $\tilde{b}_{(0)}^{a a}=\tilde{b}_{(1)}^{a a}=1, v_{F}=1$, $X=(\tau, x), Y=\left(\tau^{\prime}, y\right)$ and $P=\left(\omega_{n}, k_{1}\right)$ we get

$$
B_{ \pm \beta}^{\text {Thirring }}\left(x, \tau, y, \tau^{\prime}\right)=\exp \left\{-\frac{2 \pi}{\beta} \frac{\left(\frac{g^{2}}{\pi}\right)^{2}}{\left(1+\frac{g^{2}}{\pi}\right)} \sum_{n=-\infty}^{\infty} \int \frac{d p}{2 \pi} \frac{\operatorname{sen}^{2} \frac{P \cdot(X-Y)}{2}}{P^{2}}\right\}
$$

which, together with the corresponding free fermion contribution, can be easily shown to coincide with the well-known result for the local and covariant case $[12,7]$.

\section{Momentum distribution}

Once we have the fermionic two-point functions, it will be rather simple to get a closed expression for the electronic momentum distribution at finite temperature. Let us consider electrons with spin-up, whose distribution at finite temperature is given by

$$
N_{ \pm \uparrow}(q)=i \int_{-\infty}^{+\infty} d z_{1} e^{-i q z_{1}} \lim _{z_{0} \rightarrow 0} G_{ \pm \uparrow}^{\beta}\left(z_{0}, z_{1}\right)
$$

where $Z=\left(z_{0}, z_{1}\right)=X-Y$.

Going back to $(4.5)$ we can take the limit $\left(\tau-\tau^{\prime}\right) \rightarrow 0,\left(z_{0} \rightarrow 0\right)$ in $G_{ \pm}^{(0) \beta}$ and perform the sum over $n$. We obtain

$$
\lim _{z_{0} \rightarrow 0} G_{ \pm \uparrow}^{(0) \beta}\left(z_{0}, z_{1}\right)=\frac{1}{4 \pi} e^{ \pm i i_{F} p_{F} z_{1}} \int_{-\infty}^{+\infty} d k_{1} e^{-i k_{1} z_{1}} \cot \left(\frac{\pi}{2} \mp \frac{i \beta k_{1} v_{F}}{2}\right) .
$$


Evaluating the $k_{1}$-integral we arrive at

$$
\lim _{z_{0} \rightarrow 0} G_{ \pm \uparrow}^{(0) \beta}\left(z_{0}, z_{1}\right)=\mp \frac{\pi}{2 \beta v_{F}} e^{ \pm i v_{F} p_{F} z_{1}} \operatorname{cosech}\left(\frac{\pi^{2} z_{1}}{\beta v_{F}}\right) .
$$

Using this result together with the bosonic factors computed in the previous section, the momentum distribution can be expressed as

$$
\begin{aligned}
N_{ \pm \uparrow}(q)= & \mp \frac{i \pi}{2 \beta v_{F}} \int_{-\infty}^{+\infty} d z_{1} e^{-i z_{1}\left(q \mp i_{F} p_{F}\right)} \operatorname{cosech}\left(\frac{\pi^{2} z_{1}}{\beta v_{F}}\right) \\
& \times \exp \left\{\frac{-8}{\pi} \int d k_{1} \sin ^{2} \frac{k_{1} z_{1}}{2}\left(I_{ \pm}^{00}+I_{ \pm}^{11}\right)\left(k_{1}\right)\right\},
\end{aligned}
$$

where we have defined $I_{ \pm}^{i i}=\sum_{n=-\infty}^{\infty}\left[B_{n}^{i i}-A_{n}^{i i} \mp i C_{n}^{i i}\right] / \Delta_{n}^{i i}$. At this point it is interesting to observe that for the physically relevant case in which the potentials are temperatureindependent, the sum can be readily evaluated, yielding

$$
\begin{aligned}
I_{ \pm}^{i i}\left(k_{1}\right)= & \frac{-\beta^{2}\left(v_{F} \lambda+\tilde{b}_{(1)}^{i i} v_{F}^{-2}-\tilde{b}_{(0)}^{i i}\right)}{16 \pi^{2} \tilde{b}_{(0)}^{i i}\left(v_{F} \lambda+\tilde{b}_{(1)}^{i i}\right)} \frac{v_{F}^{2} \pi}{\left(T^{i i}\right)^{2}-S^{2}} \\
& \times\left\{\left(S-R^{i i}\right) \cot (\pi S)-\left(\frac{\left(T^{i i}\right)^{2}-R^{i i} S}{T^{i i}}\right) \cot \left(\pi T^{i i}\right)\right\}
\end{aligned}
$$

where

$$
\begin{aligned}
R^{i i}\left(k_{1}\right) & =\frac{i k_{1} \beta v_{F}}{2 \pi} \frac{\lambda+v_{F} \tilde{b}_{(0)}^{i i}-\tilde{b}_{(1)}^{i i} v_{F}^{-1}}{\lambda+\tilde{b}_{(1)}^{i i} v_{F}^{-1}-v_{F} \tilde{b}_{(0)}^{i i}}, \\
S\left(k_{1}\right) & =\frac{i k_{1} \beta v_{F}}{2 \pi}, \\
T^{i i}\left(k_{1}\right) & =\frac{i k_{1} \beta}{2 \pi}\left[\frac{\tilde{b}_{(1)}^{i i}}{\tilde{b}_{(0)}^{i i}}\left(\frac{v_{F}^{-1} \lambda+\tilde{b}_{(0)}^{i i}}{v_{F} \lambda+\tilde{b}_{(1)}^{i i}}\right)\right]^{1 / 2} .
\end{aligned}
$$

Thus, we finally get

$$
\begin{aligned}
N_{\uparrow}(q)= & N_{+\uparrow}(q)+N_{-\uparrow}(q)=\frac{\pi}{\beta v_{F}} \int_{-\infty}^{+\infty} d z_{1} e^{-i q z_{1}} \sin \left(p_{F} z_{1}\right) \\
& \times \operatorname{cosech}\left(\frac{\pi^{2} z_{1}}{\beta v_{F}}\right) \exp \left\{\frac{-8}{\pi} \int d k_{1} \sin ^{2} \frac{k_{1} z_{1}}{2}\left(I_{+}^{00}+I_{+}^{11}\right)\left(k_{1}\right)\right\} .
\end{aligned}
$$

Of course, in order to have the complete result one has to add the contribution corresponding to spin-down electrons. In the present situation (no spin-flipping interactions) it is easy to see that both up and down results coincide and therefore, (5.9) must be multiplied by 2 .

This result could be used to perform quantitative studies of the momentum distribution in the context of 1d many-body systems [13], as functional of potentials. In fact, this 
formula is the extension to the $T \neq 0$ case of the one first presented in [4]. As such, it could be helpful in order to verify if the interplay between electron-electron interactions and thermal corrections could give rise to a restoration of Fermi-liquid behavior, as suggested in the literature $[14,15]$.

\section{Conclusions}

In this work we have extended a recently proposed approach to the bosonization of a Thirring model with non-local current-current interaction, originally developed at $T=0$ [4], to the finite-temperature case. In view of the connection between this model and a one-dimensional system of fermions (in fact our non-local model coincides with the Tomonaga-Luttinger model for a particular choice of the coupling potentials), the present study is relevant not only from a purely academic point of view but also as a possible starting point to examine thermodynamical properties of a Luttinger liquid through an alternative field-theoretical formulation.

In Section 2 we have shown how the standard techniques of finite temperature local and covariant QFT's [5,6,11] can be also successfully implemented in the non-local Thirring model. We obtained an expression for the partition function as functional of the forward-scattering potentials. From this result, in Section 3 we derived the corresponding formulae for the Helmholtz free energy, the energy and the specific heat. In Sections 4 and 5 we computed the two-point fermionic correlation and the momentum distribution, respectively.

One interesting aspect of our results is that they provide practical formulae to check the validity of different potentials. Moreover, they could be used to explore the interplay between thermal effects and electron-electron scattering (through suitable potentials). However, in order to make these studies more realistic one should include two ingredients that were disregarded in this paper: spin-flippings and backward-scattering. We hope to report on some of these issues in the close future.

\section{Acknowledgements}

The authors are financially supported by Universidad Nacional de La Plata (UNLP) and Consejo Nacional de Investigaciones Científicas y Técnicas (CONICET), Argentina.

\section{References}

[1] J. Voit, Rep. Prog. Phys. 58 (1995) 977.

[2] F. Haldane, J. Phys. C 14 (1981) 2585.

13] Tomonaga, Prog. Theor. Phys. 5 (1950) 544;

J. Luttinger, J. Math. Phys. 4 (1963) 1154.

E. Lieb and D. Mattis, J. Math. Phys. 6 (1965) 304. 
[4] C.M. Naón, M.C. von Reichenbach and M.L. Trobo, Nucl. Phys. [FS] B 435 (1995) 567; D.G.Barci and C.M.Naón, Int. J. Mod. Phys. A 13 (1998) 1169.

[5] C. Bernard, Phys. Rev. D 9 (1974) 3312.

[6] T. Matsubara, Prog. Theor. Phys. 14 (1955) 351.

[7] M.V. Manías, C.M. Naón and M.L. Trobo, Phys. Lett. B 416 (1998) 157, and references therein.

[8] B. Klaiber, Lectures in Theoretical Physics, Boulder, Colorado, 1967, Vol.10 A, ed. A. Barut and W. Brittin (Gordon and Breach, New York) p. 141.

[9] J. Sólyom, Adv. Phys. 28 (1979) 201.

[10] M. Reuter and W. Dittrich, Phys. Rev. D 32 (1985) 513.

[11] L. Dolan and R. Jackiw, Phys. Rev. D. 9 (1974) 3320.

[12] F. Ruiz-Ruiz and R.F. Alvarez-Estrada, Phys. Rev. D 35 (1987) 3161.

[13] H. Gutfreund and M. Schick, Phys. Rev. 168 (1968) 418.

[14] B. Hu and S. Das Sarma, Phys. Rev. B 48 (1993) 5469.

[15] C.M. Naón, M.C. von Reichenbach and M.L. Trobo, Nucl. Phys. [FS] B 485 (1997) 665. 